\title{
Identification of Cyclase-Associated Protein-2 as a Novel Biomarker for Early- Stage Hepatocellular Carcinoma
}

\author{
Mohammed Amin Mohammed ${ }^{1 *}$, Nesreen Moustafa Omar², Soad Amin Mohammed ${ }^{3}$ and Ahmed \\ Galal Deiab ${ }^{1}$ \\ ${ }^{1}$ Faculty of Medicine, Department of Internal Medicine, Gastroenterology, Hepatology and Endoscopy Unit, Mansoura University, Egypt \\ ${ }^{2}$ Faculty of Medicine, Department of Histology and Cell Biology, Mansoura University, Egypt \\ ${ }^{3}$ Faculty of Pharmacy, Department of Microbiology, Al-Azhar University, Egypt
}

${ }^{*}$ Corresponding author: Mohammed Amin Mohammed, Faculty of Medicine, Department of Internal Medicine, Gastroenterology, Hepatology and Endoscopy Unit, Mansoura University, Egypt, Tel: 00201122022900; E-mail: liver2345@gmail.com; liver2003@mans.edu.eg

Rec date: July 18, 2017; Acc date: September 19, 2017; Pub date: September 22, 2017

Citation: Mohammed MA, Omar NM, Mohammed SA, Deiab AG (2017) Identification of Cyclase-Associated Protein-2 as A Novel Biomarker for Early-Stage Hepatocellular Carcinoma. J Clin Gastroenterol Hepatol Vol.1 No.3:26.

\section{Abstract}

Background: Hepatocellular carcinoma (HCC) is an aggressive deadly cancer with few therapeutic options mostly limited for early-stage HCC. Unfortunately, alphafetoprotein (AFP) has a limited performance, especially in early-stage HCC.

Objectives: to investigate plasma levels of Cyclaseassociated protein-2 (CAP2) as a new biomarker and evaluate its role in detecting early-stage and AFP-negative HCC Egyptian patients.

Methods: Plasma CAP2 and AFP levels in $150 \mathrm{HCC}, 150$ cirrhotic patients, 150 healthy controls. Correlation with tumor behavior, the area under the curve (AUC), sensitivity, specificity, and diagnostic accuracy were analyzed.

Results: Plasma CAP2 and AFP levels were significantly elevated in HCC patients than liver cirrhosis and controls. Only plasma CAP2 levels significantly correlated with clinico-pathological characteristics of HCC (BCLC, histological and clinical stages) but not correlated with patient's age, gender, viral infection status or AFP levels. Compared to AFP, CAP2 had significantly higher AUC: 0.86 (0.79-0.93) vs. 0.75 (0.65-0.85), Sensitivity: $81.5 \%$ vs. $62 \%$ in all HCCs and significantly higher AUC: $0.80(0.72-0.89)$ vs. $0.68(0.58-0.79$, Sensitivity: $80.5 \%$ vs. $43.1 \%$ in earlystage HCC. Moreover, the combined diagnostic value of both CAP2+AFP was statistically significantly better than either CAP2 or AFP alone. Also, CAP2 could predict $82.4 \%$ of AFP-negative HCCs [AUC: 0.85 (0.77-0.92)] and 73.5\% of AFP-negative early-stage HCCs [AUC: $0.80(0.72-0.88)$ ].

Conclusion: Compared with AFP, CAP2 was significantly elevated in HCC patients with higher sensitivity and AUC especially for early-stage HCC. Moreover, CAP2 was significantly correlated with the clinico-pathological features of HCC. CAP2 could be a novel biomarker predicting early-stage, AFP-negative, and AFP-negative early-stage HCC patients.

Keywords: Alpha-fetoprotein-negative HCC; BCLC stage; Biomarkers; Cyclase-associated protein-2; Hepatocellular carcinoma

\section{Introduction}

Hepatocellular carcinoma (HCC) is considered the fifth commonest cancer in men, the seventh most common cancer in women worldwide and the second leading cause of cancer death worldwide with more than 1.6 million annual deaths $[1,2]$. HCC is one of the most aggressive cancers with limited therapeutic options and more than half a million people worldwide achieve HCC diagnosis [3,4]. The commonest and strongest risk factor for HCC development is liver cirrhosis; more than $90 \%$ of HCCs develop on top of a cirrhotic liver due to chronic hepatitis $B$, hepatitis $C$, alcoholic steatohepatitis, nonalcoholic steatohepatitis, diabetes or obesity [5]. Patients with advanced HCC progressing to the terminal stage have less than $10 \%$ one-year survival rate. In last decades, despite the recent considerable advances in surgical and radiological interventions of HCC and the clinical implementation of many therapeutic modalities, only a poor improvement in the fiveyear survival has been observed worldwide [6]. Hepatocarcinogenesis is usually silent and HCC patients usually experience symptoms in advanced disease stage. HCC prognosis depends markedly on its stage at the time of diagnosis. Unfortunately, Sorafenib which is a multikinase inhibitor and the only FDA-approved drug for advanced HCC has limited survival benefits [7]. Moreover, the curative treatments of HCC are largely limited to early disease stage. The recent therapeutic strategies including surgical resection, transarterial chemoembolization, percutaneous intervention (radiofrequency ablation and ethanol injection) or even orthotopic liver transplantation are effective only at an earlystage of $\mathrm{HCC}$ with approximately $70 \%$ recurrence within five years [8]. Thus, the early detection of HCC is an important and 
crucial goal for all researchers. The early diagnosis and prognostic prediction of $\mathrm{HCC}$ are much difficult due to the coexistence of liver cirrhosis and inflammation in HCC [9]. Unfortunately, alpha fetoprotein (AFP) which was the golden marker of HCC lacks both sensitivity and specificity, has limited performance especially in early-stage HCCs and is no longer recommended by international surveillance guidelines [10].

Given these data, much interest was given for developing new potential therapeutic strategies. Also, the discovery of ideal novel biological non-invasive diagnostic biomarkers with a high-performance and prognostic prediction for this aggressive deadly disease has become a major focus of cancer research. The ideal biomarker should be protein-, RNA-, DNA-, or antibody-based, measurable in serum or urine, affordable, ethnically-specific and practically deployable in both the developed and developing worlds. Recently, a wide variety of novel biomarkers has been suggested to reinforce the current surveillance methods and predict early-stage HCC in patient's at-risk [11].

\section{Serum biomarkers of HCC includes}

Alpha-fetoprotein (AFP), Lens culinaris agglutinin-reactive AFP (AFP-L3), des-gamma-carboxy prothrombin (also known as prothrombin induced by vitamin $\mathrm{K}$ absence II, PIVKA II), AlphaI-fucosidase (AFU), Glypican-3 (GPC3), Vascular endothelial growth factor, Interleukin-8, Transforming growth factor-beta 1, Tumor-specific growth factor, serine protease inhibitor squamous cell carcinoma antigen-immunoglobulin $\mathrm{M}$ complex (SCCA-IGM), Heat shock proteins (HSP70), Annexin I (ANX1), cyclase-associated protein (CAP), microRNAs (miRNAs), Exosomes, Osteopontin (OPN), Eag1 channels and Serum metabolites (Lysophosphatidylcholines, Free Fatty Acids species, serum bile acids) Urinary biomarkers of HCC include: Nucleosides, TGF $\alpha$ and $\beta$, Neopterin, Polyamines, Urinary trypsin inhibitor, soluble urinary metabolites [12-14].

The Cyclase-associated protein (CAP), an evolutionary highly conserved multifunctional actin-binding protein consisting of 474 to 551 amino acid residues, is present in mammals and a wide range of organisms including yeast, flies, and plants. It is involved in and plays a crucial role in species-specific signaling pathways $[15,16]$. CAP activates adenylyl cyclase, binds to Gactin mediating the dynamics of actin polymerization and is required for normal cellular morphology, locomotion, division, endocytosis, growth, and development [17]. In higher eukaryotes, two different homologs (CAP1 and CAP2), are present and share about $76 \%$ amino acid similarity. CAP2 is rarely present in a few of tissues and co-localized with actin in skeletal muscle cells $[18,19]$. During mice embryos' development, CAP2 was identifiable throughout cardiogenesis and its depletion led to dilated cardiomyopathy and other cardiac detects [20]. In human, CAP2 was detectable and differently expressed in many cancers. For example, CAP2 mRNA has been reported to be highly upregulated in thyroid, kidney, bladder and breast cancers and down-regulated in breast fibroadenoma [21].

Also, CAP2 was involved in hepatic carcinogenesis being over-expressed in HCC patients demonstrated by immunohistochemistry staining [22]. However, the data in literature describing its relevant prognostic implication in HCC are so far limited and elusive. Moreover, it is not clear if plasma level of CAP2 might be detected or not. The relationship between plasma levels of CAP2 and AFP levels in different stages of HCC is also not known. Thus, the objective of this study was to investigate the plasma levels of CAP2 and AFP in HCC, cirrhosis, healthy subjects. Also, we evaluated its role in detecting early-stage and AFP-negative HCC Egyptian patients.

\section{Patients and Methods}

\section{Subjects}

A total of 300 adult consecutive outpatient subjects with a confirmed diagnosis of liver cirrhosis of any etiology (150) and HCC of any stage (150) and receiving long-term follow-up were initially enrolled in this study. The age range was 30-65 years and male/female ratio was 1.36 (259/191). Another one hundred fifty (150) age- and sex-matched healthy control subjects were also enrolled. The study was initiated in the January 2015 and continued through 2017. The study was approved by the Ethical Commission and Institutional review board of Mansoura Faculty of Medicine in Egypt (MFM-IRB; Code No: R/16.02.91). A written informed conscious consent was obtained from all participants before their participation. The inclusion criterion was the diagnosis of liver cirrhosis and HCC. Exclusion criteria were an age below 18 years and over 70 years, a history of another cancer of any type within the last 5 years, a history of solid organ transplantation or previous bone marrow transplantation, and local or systemic tumorspecific treatment within the last month. Patients with chronic renal failure, bone disorders, thyroid disorders, cardiac failure (ejection fraction $<50 \%$ ), and systemic bacterial or fungal infection were excluded from the present study.

\section{Methods}

Initially, all participants completed a detailed questionnaire regarding diet and habits and submitted to thorough history taking with detailed physical examinations and relevant medical history. At the day of study inclusion, three milliliters of venous blood (by venipuncture of the antecubital vein) were obtained from all participants and the serum samples were centrifuged at $3000 \mathrm{rpm}$ then aliquoted and stored at $-70^{\circ} \mathrm{C}$ until assayed. Laboratory parameters, Ultrasound, CT scans and MRI imaging, the model of end-stage liver disease (MELD score) and Child-Pugh scores were assessed at the time of inclusion in the study [23].

Liver cirrhosis: Liver cirrhosis was diagnosed by ascites, esophageal varices, fundic varices, splenomegaly, jaundice, imaging and liver biopsies (if available, according to modifiedknodell histological activity index) [24].

HCC: HCC was diagnosed by 4-phase multi-detector computed tomography (CT) scan, dynamic contrast-enhanced magnetic resonance imaging (MRI) $[25,26]$. Diagnosis of HCC was confirmed if there is one of the following three items: 
- One or more of liver nodules $>1 \mathrm{~cm}$ in diameter in $\mathrm{CT}$ or MRI.

- Early arterial enhancement with $\alpha$-fetoprotein $\geq 400$ $\mathrm{ng} / \mathrm{mL}$.

- Typical features of dynamic imaging (arterial phase hypervascularity and washout in portal venous or delayed phases) regardless $\alpha$-fetoprotein level.

Barcelona Clinic Liver Cancer (BCLC): The Barcelona Clinic Liver Cancer (BCLC) staging system was used to determine the stage of HCC. BCLC staging has been authenticated by liver expert groups (EASL and AASLD) as it allocates stage-specific management options, predicts survival and is likely to be updated. BCLC staging is best validated and suited for selection of early-stage HCC patients who could benefit from curative therapies [27].

- Very early-stage HCC or BCLC stage 0 refers to patients with single lesion $\leq 2 \mathrm{~cm}$ in diameter or carcinoma in situ without vascular involvement or metastasis.

- Early-stage HCC or BCLC stage A refers to patients with single or up to three nodules of $\leq 3 \mathrm{~cm}$ in diameter each, without portal vein thrombosis or extra-hepatic metastasis.

- The intermediate stage HCC or BCLC stage B represents asymptomatic, large, or multifocal HCCs without evidence of vascular invasion or extra-hepatic metastasis.

- Advanced and Late stages HCC or BCLC stage Cand D constitutes symptomatic patients with vascular invasion or extra-hepatic metastasis.

All participants were assigned to the following groups:

- Control group: It comprised 150 healthy controls (Age: 56.3 \pm 1.21 years; Males/ Females: 91/59).

- Liver Cirrhosis group (LC): It comprised 150 cirrhotic patients (Age: $57.15 \pm 1.02$ years; Males/ Females: 88/62).

- All HCC group: It comprised 150 patients with HCC at any stage (Age: $62.0 \pm 5.39$ years; Males/ Females: 33/35). This group included (Table 3 ).

- Sixty-eight patients with early-stage HCC (Age: $59.3 \pm 2.1$ years; Males/ Females: 27/29)

- Fifty-six patients with AFP-ve HCC (Age: $59.3 \pm 2.1$ years; Males/ Females: 27/29) of which, fifty patients were AFPve early stage HCC (Age: $58.3 \pm 2.2$ years; Males/ Females: 24/26).

\section{Collection of blood samples}

At the day of study inclusion, three milliliters of venous blood (by venipuncture of the antecubital vein) were obtained from all participants and the serum samples were centrifuged at $3500 \mathrm{rpm}$ for 15 minutes at room temperature then aliquoted and stored at $-70^{\circ} \mathrm{C}$ until assayed for AFP and CAP2. Avoid repeated freeze/thaw cycles.

\section{Plasma AFP and CAP2 Protein Concentration Assay}

Plasma levels of CAP2 and AFP were measured using enzyme-linked immunosorbent assay (ELISA) kits purchased from Rand D systems (Minneapolis, MN, USA). The human Adenylyl Cyclase associated protein 2 (CAP2) ELISA KIT is a ready-to-use microwell, strip plate ELISA Kit for analyzing the presence of the CAP2 in biological samples. The sensitivities of ELISA kit of CAP2 and AFP were $0.037 \mathrm{ng} / \mathrm{mL}$ and $0.046 \mathrm{ng} / \mathrm{mL}$ respectively. The assay range was $0.300-20 \mathrm{ng} / \mathrm{mL}$ for CAP2 and $0.312-20 \mathrm{ng} / \mathrm{mL}$ for AFP.

The Assay principle applied in the kits is Sandwich enzyme linked immunosorbent assay. The ELISA analytical biochemical technique of the kit is based on CAP2 antibody-CAP2 antigen interactions (immuno-sorbent) and a Horseradish Peroxidase (HRP) colorimetric detection system to detect CAP2 antigen targets in samples. The microtiter plate provided in the kit has been pre-coated with an antibody specific to Adenylyl Cyclase Associated Protein 2 (CAP2). Standards or samples are then added to the appropriate microtiter plate wells with a biotinconjugated antibody specific to Adenylyl Cyclase Associated Protein 2 (CAP2). Next, Avidin conjugated to HRP is added to each microplate well and incubated. After TMB substrate solution is added, only those wells that contain adenylyl cyclase associated protein-2 (CAP2), biotin-conjugated antibody and enzyme-conjugated avidin will exhibit a change in color. The enzyme-substrate reaction is terminated by the addition of sulphuric acid solution and the color change is measured spectrophotometrically at a wavelength of $450 \mathrm{~nm} \pm$ $10 \mathrm{~nm}$. The concentration of adenylyl cyclase associated protein-2 (CAP2) in the samples is then determined by comparing the optical densities (OD) of the samples to the standard curve.

\section{Assay procedure}

Plasma CAP2 and AFP concentrations were measured according to manufacturer's recommendation. Briefly, $10 \mu \mathrm{L}$ of plasma samples were mixed with $40 \mu \mathrm{L}$ of sample dilution buffer and incubated in 96-well plates coated with antibodies for $30 \mathrm{~min}$ at $37^{\circ} \mathrm{C}$. The solutions were decanted followed by washing. Then, $50 \mu \mathrm{L}$ HRP-conjugated secondary antibodies were added into the wells and incubated for $30 \mathrm{~min}$. After washing, $50 \mu \mathrm{L}$ of each chromogen solution $\mathrm{A}$ and chromogen solution $B$ were added into the wells, and incubated for $15 \mathrm{~min}$ at $37^{\circ} \mathrm{C}$. The reaction was stopped by adding $50 \mu \mathrm{L}$ of stop solution. The $O D$ values were determined in 96-well plate reader (Bio-Rad Laboratories, Hercules, MA, USA) at $450 \mathrm{~nm}$ wave length. All tests were performed in duplicate, average OD values were calculated and the plasma levels of CAP2 and AFP were determined by standard curve. Data were collected and analyzed, intra-batch variation was controlled within $5 \%$, and inter-batch variation was less $10 \%$. 


\section{Statistical Analysis}

Data were analyzed using SPSS software (Version 17.0, SPSS Inc., Chicago; IL). Quantitative (continuous) data were expressed as (Mean \pm Standard Deviation) while qualitative data and categorical variables were expressed as number and percentage. Categorical variables were compared using the chi-square $\left(\chi^{2}\right)$ test or Fisher's exact test. Subgroups were compared using the Mc-Nemar test. Comparisons between the groups were performed using the Student's t-test whenever applicable. Mann-Whitney U-test was used for the continuous ordinal data between two qualitative variables. One Way Analysis of Variance (ANOVA) compares more than two groups. Correlations between markers and other variables were evaluated using the Spearman rank correlation coefficient test. Variables that achieved statistical significance with the univariate analysis were included in multiple regression analysis to differentiate HCC patients from cirrhotic patients and to evaluate the independent factors associated with high CAP2 or AFP. Receiver operating characteristic curves (ROC) was constructed to determine optimal cutoff values, diagnostic accuracy and performance of CAP2 and AFP. Area under the curve (AUC) calculations and their 95\% confidence intervals were used to evaluate diagnostic values [28]. For all statistical studies, $\mathrm{P}<0.05$ was considered to be statistically significant. Sensitivity, specificity, and predictive values were calculated to study the overall predictability according to the following equations: [29]
- Positive (+ve) predictive value $=($ No of true + ve cases $/$ NO of all +ve cases with screening test $) \times 100$.

- Negative (-ve) predictive value $=($ No of true - ve cases $/$ NO of all -ve cases with screening test) $\times 100$.

- Sensitivity=(No of true +ve cases/No of all +ve cases with reference test) $\times 100$.

- Specificity=(No of true -ve cases/No of all -ve cases with reference test) $\times 100$.

- Overall predictability (accuracy) $=($ No of true +ve and true ve cases/ total No of all +ve and all-ve cases) $\times 100$.

\section{Results}

The patient basic demographic, clinical and laboratory data in all studied groups and subgroups were shown in Table 1. When compared with control or LC groups, patients with HCC had a statistically significantly higher mean age, serum AST, serum ALT, serum Bilirubin, INR, plasma AFP and CAP2 levels and had a significantly lower serum albumin and platelet counts $(P<0.05$ for all). Nevertheless, patients with early-stage HCC had no significant differences in AFP levels when compared with LC patients $(P<0.05)$. Chronic Hepatitis $C$ was the commonest etiology of liver cirrhosis in either HCC or nonHCC patients $(63.3 \%$ and $62 \%$ respectively) followed by Chronic Hepatitis B ( $20 \%$ and $18.7 \%$ respectively) however, there were no significant differences in plasma levels of either CAP2 or AFP in chronic hepatitis B or C.

Table 1: The demographic, clinical and laboratory characteristics of patients in the studied groups.

\begin{tabular}{|c|c|c|c|c|c|c|c|}
\hline Variables & $\begin{array}{l}\text { Control } \\
(150)\end{array}$ & LC (150) & HCC (150) & $\begin{array}{l}\text { Early HCC } \\
(68)\end{array}$ & $\begin{array}{l}\text { AFP-ve HCC } \\
(56)\end{array}$ & $\begin{array}{l}\text { AFP-ve early-stage } \\
\text { HCC (50) }\end{array}$ & $\begin{array}{l}\text { ANOV } \\
\text { A }\end{array}$ \\
\hline Age (Years) & $56.3 \pm 1.21$ & $\begin{array}{ll}57.15 & \pm \\
1.02 & \end{array}$ & $62.0 \pm 5.39$ & $61.4 \pm 4.39$ & $59.3 \pm 2.1$ & $58.3 \pm 2.2$ & 0.041 \\
\hline Male /Female & $80 / 70$ & $91 / 59$ & $88 / 62$ & $33 / 35$ & $27 / 29$ & $24 / 26$ & 0.347 \\
\hline $\begin{array}{l}\text { Etiology: [HCV/HBC/Alcoholism/ } \\
\text { others] }\end{array}$ & - & $93 / 30 / 8 / 19$ & $95 / 28 / 8 / 19$ & $36 / 16 / 3 / 13$ & $35 / 11 / 2 / 8$ & $33 / 9 / 2 / 6$ & - \\
\hline AST (U/L) & $39.6 \pm 1.9$ & $99.8 \pm 3.17$ & $113.1 \pm 2.08$ & $109.1 \pm 3.78$ & $101.8 \pm 3.2$ & $103.8 \pm 3.2$ & 0.005 \\
\hline ALT (U/L) & $41.45 \pm 1.6$ & $86.0 \pm 1.7$ & $117.65 \pm 3.8$ & $113.6 \pm 5.8$ & $89.0 \pm 1.6$ & $90.0 \pm 1.7$ & 0.006 \\
\hline $\begin{array}{l}\text { S. bilirubin } \\
(\mathrm{mg} / \mathrm{dL})\end{array}$ & $1.2 \pm 0.1$ & $1.3 \pm 0.2$ & $2.8 \pm 0.8$ & $2.4 \pm 0.8$ & $1.2 \pm 0.3$ & $1.3 \pm 0.3$ & 0.012 \\
\hline Serum Albumin (g/dL) & $4.6 \pm 1.1$ & $2.9 \pm 1.9$ & $2.1 \pm 2.1$ & $2.2 \pm 2.1$ & $2.7 \pm 1.3$ & $2.6 \pm 1.3$ & 0.034 \\
\hline S. Creatinine $(\mathrm{mg} / \mathrm{dL})$ & $0.9 \pm 0.4$ & $1.1 \pm 0.5$ & $1.2 \pm 0.4$ & $1.2 \pm 0.2$ & $1.1 \pm 0.8$ & $1.1 \pm 1$ & 0.071 \\
\hline Platelets $(104 / \mu \mathrm{L})$ & $22.6 \pm 0.23$ & $14.49 \pm 0.5$ & $10.78 \pm 0.6$ & $11.78 \pm 0.6$ & $12.1 \pm 0.6$ & $11.1 \pm 0.6$ & 0.001 \\
\hline INR & $1.1 \pm 0.1$ & $1.3 \pm 0.2$ & $1.7 \pm 0.4$ & $1.7 \pm 0.4$ & $1.2 \pm 0.3$ & $1.2 \pm 0.5$ & 0.023 \\
\hline AFP (ng/mL) & $4.7 \pm 3.1$ & $40.8 \pm 62.3$ & $\begin{array}{ll}1016.8 & \pm \\
946.1 & \end{array}$ & $33.3 \pm 7.1$ & $12.8 \pm 2.8$ & $126 \pm 2.6$ & $<0.001$ \\
\hline Plasma CAP2 levels & $4.44 \pm 3.3$ & $7.53 \pm 3.54$ & $37.1 \pm 8.8$ & $34.1 \pm 10.2$ & $31.7 \pm 10.1$ & $31.9 \pm 9.9$ & $<0.001$ \\
\hline \multicolumn{8}{|c|}{$\begin{array}{l}\text { Data were expressed as } \mathrm{M} \pm \mathrm{SD} \text { : Mean } \pm \text { Standard Deviation; INR: International Normalized Ratio; LC: Liver Cirrhosis; HCC: Hepatocellular Carcinoma; AST: } \\
\text { Aspartate Transaminase; ALT: Alanine Transaminase; AFP: Serum Alpha-Fetoprotein Level (ng/mL); CAP2: Adenylyl Cyclase-Associated Protein-2 (ng/mL); HBV: } \\
\text { Hepatitis B Virus; HCV: Hepatitis C Virus; P: Probability; ANOVA: One Way Analysis of Variance; }(\mathrm{P}<0.05 \text { was considered to be statistically significant). }\end{array}$} \\
\hline
\end{tabular}


The clinico-pathological characteristics of patients with HCC and $L C$ and their relations to the plasma CAP2 levels were demonstrated in Table 2. In HCC patients, Higher plasma CAP2 levels were significantly related associated advanced BCLC stages of HCC, large tumor nodules, the presence of either metastasis or portal vein thrombosis $(\mathrm{P}=0.001)$ but not with age, gender or etiological cause $(P>0.05)$. Moreover, high plasma CAP2 levels were significantly associated with high Child Pugh scores in HCC patients with underlying cirrhosis $(p=0.0001)$.

Table 2: Comparisons between plasma levels of CAP2 in different clinicopathological characteristics of patients with HCC and LC.

\begin{tabular}{|c|c|c|c|c|c|c|c|}
\hline \multicolumn{2}{|l|}{ Variables } & \multirow{2}{*}{$\begin{array}{l}\text { CAP2 HCC } \\
\mathrm{N}(\%)\end{array}$} & \multirow[b]{2}{*}{$M \pm S D$} & \multirow[b]{2}{*}{ P-value } & \multicolumn{3}{|l|}{ CAP2 LC } \\
\hline & & & & & N (\%) & $M \pm S D$ & P-value \\
\hline \multirow[t]{2}{*}{ Age } & $\geq 50$ years & $102(68)$ & $36.1 \pm 7.5$ & \multirow[t]{2}{*}{0.059} & $92(61.3)$ & $7.6 \pm 3.3$ & \multirow[t]{2}{*}{0.258} \\
\hline & $<50$ years & $48(32)$ & $34.4 \pm 10.2$ & & $58(38.7)$ & $6.9 \pm 3.4$ & \\
\hline \multirow[t]{2}{*}{ Gender } & Male & $88(58.7)$ & $34.1 \pm 9.8$ & & $91(60.7)$ & $7.1 \pm 3.6$ & \\
\hline & Female & $62(41.3)$ & $36.2 \pm 7.9$ & 0.092 & $59(39.3)$ & $7.6 \pm 3.4$ & 0.671 \\
\hline \multirow[t]{4}{*}{ Etiology } & $\mathrm{HCV}$ & $95(63.3)$ & $34.2 \pm 9.8$ & & $93(62)$ & $7.4 \pm 3.5$ & \\
\hline & HBV & $28(18.7)$ & $34.6 \pm 8.8$ & \multirow[t]{3}{*}{0.607} & $30(20)$ & $7.7 \pm 3.4$ & \multirow[t]{3}{*}{0.302} \\
\hline & Alcoholism & $8(5.3)$ & $30.8 \pm 10.6$ & & $8(5.3)$ & $10.2 \pm 3.3$ & \\
\hline & Others & $19(12.7)$ & $35.3 \pm 7.1$ & & $19(12.7)$ & $5.9 \pm 3.7$ & \\
\hline \multirow[t]{2}{*}{ Metastasis } & No & $122(81.3)$ & $31.8 \pm 9.1$ & \multirow[t]{2}{*}{$0.001 a$} & - & - & \\
\hline & Yes & $28(18.7)$ & $40.6 \pm 5.2$ & & - & - & \\
\hline \multirow[t]{2}{*}{ PVT } & No & $108(72)$ & $30.9 \pm 8.4$ & \multirow[t]{2}{*}{$0.001 a$} & - & - & \\
\hline & Yes & $42(28)$ & $41.5 \pm 5.8$ & & - & - & \\
\hline \multirow[t]{3}{*}{ Tumor size } & $\geq 2 \mathrm{~cm}$ & $54(36)$ & $28.6 \pm 8.9$ & \multirow[t]{2}{*}{$0.001 \mathrm{~b}$} & - & - & \\
\hline & $>2, \leq 5 \mathrm{~cm}$ & $56(37.3)$ & $37.2 \pm 8.1$ & & - & - & \\
\hline & $>5 \mathrm{~cm}$ & $40(26.7)$ & $36.8 \pm 7.6$ & & - & - & \\
\hline \multirow[t]{3}{*}{ BCLC stage } & $0, A$ & $68(45.3)$ & $35.1 \pm 10.2$ & \multirow[t]{3}{*}{$0.001 b$} & - & - & \\
\hline & $\mathrm{B}, \mathrm{C}$ & $70(46.7)$ & $37.1 \pm 7.2$ & & - & - & \\
\hline & D & $12(8)$ & $38 \pm 7.5$ & & - & - & \\
\hline \multirow[t]{4}{*}{ Child Pugh } & No LC & $49(32.7)$ & $29.2 \pm 9.5$ & \multirow[t]{4}{*}{$0.0001 b$} & - & - & - \\
\hline & A & $59(39.3)$ & $34.3 \pm 9.1$ & & $70(46.7)$ & $7.8 \pm 2.8$ & \multirow[t]{3}{*}{0.206} \\
\hline & B & $30(20)$ & $36.8 \pm 8.3$ & & $51(34)$ & $7.6 \pm 3.9$ & \\
\hline & C & $12(8)$ & $39.1 \pm 8.7$ & & $29(19.3)$ & $7.9 \pm 4.1$ & \\
\hline
\end{tabular}

Also, the plasma CAP2 and AFP levels in early and advanced disease stages of both HCC and LC groups were demonstrated in Table 3. Only the plasma levels of CAP2 (but not plasma AFP levels) were significantly elevated in early-stage HCC and AFPve early-stage $H C C$ than $L C$ patients $(P<0.01)$.

The Correlations between plasma CAP2 and AFP levels and the clinico-pathological characteristics in patients with HCC and LC were demonstrated in Table 4.

Only the plasma CAP2 levels (but not plasma AFP levels) were significantly and positively correlated with BCLC stages of $\mathrm{HCC}$, tumor size, the presence of either metastasis or portal vein thrombosis $(\mathrm{P}<0.01)$ but not with age, gender. Moreover, and as shown in Figure 1, no significant correlations were found between plasma levels of CAP2 and AFP in HCC patients $(r=-136-, P=0.156)$.

The receiver operating characteristic (ROC) curves for CAP2, AFP or combined (CAP2+AFP) levels were constructed to further evaluate the diagnostic role of CAP2 in All HCC patients, early-stage HCC, and AFP-ve early-stage HCC patients (Figures 2A to 2D). Also, the diagnostic cutoff values, area under the curve (AUC), Sensitivity, Specificity, Likelihood ratio 
and predictive values of CAP2 and AFP in HCC group and its subgroups were listed in Table 5.

Table 3: Plasma CAP2 and AFP levels in the different disease stages of HCC and LC groups.

\begin{tabular}{|c|c|c|c|c|c|c|c|}
\hline HCC group & & CAP2 (ng/mL) & $\operatorname{AFP}(\mathbf{n g} / \mathrm{mL})$ & LC group & & CAP2 (ng/mL) & $\operatorname{AFP}(\mathrm{ng} / \mathrm{mL})$ \\
\hline HCC stage & $\begin{array}{l}\text { AFP status } \\
(\mathrm{N})\end{array}$ & $M \pm S D$ & $M \pm S D$ & LC stage & $\begin{array}{l}\text { AFP status } \\
\text { (N) }\end{array}$ & $M \pm S D$ & $M \pm S D$ \\
\hline \multirow[t]{4}{*}{ Early } & -ve (50) & $31.7 \pm 10.1$ & $12.8 \pm 2.8$ & \multirow[t]{3}{*}{ Early } & -ve (93) & $6.3 \pm 2.95$ & $4.1 \pm 3.5$ \\
\hline & +ve (18) & $36 \pm 9.8$ & $82 \pm 47.9$ & & +ve (6) & $6.5 \pm 2.7$ & $25.5 \pm 46.6$ \\
\hline & Total (68) & $34.1 \pm 10.2$ & $33.3 \pm 7.1$ & & Total (99) & $6.75 \pm 2.9$ & $15.1 \pm 35$ \\
\hline & P1-value & $<0.01$ & $<0.001$ & & & NS & $S$ \\
\hline \multirow[t]{3}{*}{ Advanced } & -ve (6) & $40.6 \pm 5.4$ & $13.2 \pm 2.8$ & \multirow[t]{3}{*}{ Advanced } & -ve (31) & $8.8 \pm 3.43$ & $17 \pm 3.8$ \\
\hline & +ve (76) & $38.4 \pm 10.3$ & $1769.7 \pm 726.2$ & & +ve (20) & $9.1 \pm 4.4$ & $106.5 \pm 70.8$ \\
\hline & Total (82) & $39.6 \pm 10.1$ & $1713 \pm 767$ & & Total (51) & $9 \pm 4.2$ & $90.7 \pm 72.8$ \\
\hline \multirow[t]{3}{*}{ Total } & -ve (67) & $34.4 \pm 9.8$ & $11.7 \pm 10.7$ & \multirow[t]{3}{*}{ Total } & -ve (124) & $7.56 \pm 3.1$ & $6.1 \pm 5.9$ \\
\hline & +ve (83) & $35.9 \pm 9.3$ & $1520.6 \pm 892.4$ & & +ve (26) & $7.5 \pm 3.8$ & $62.1 \pm 71.1$ \\
\hline & Total (150) & $37.1 \pm 8.8$ & $1016.8 \pm 946.1$ & & Total (150) & $7.5 \pm 3.5$ & $40.8 \pm 62.3$ \\
\hline P2-value & & $<0.01$ & $<0.0001$ & & & NS & $S$ \\
\hline \multicolumn{8}{|c|}{$\begin{array}{l}\text { Data were expressed as M } \pm \text { SD: mean } \pm \text { standard deviation; N: Number; LC: Liver Cirrhosis; HCC: Hepatocellular Carcinoma; AFP: Serum Alpha-Fetoprotein Leve } \\
\text { (Ng/MI); CAP2: Adenylyl Cyclase-Associated Protein-2 (ng/mL); -ve: Negative; +ve: Positive; HCC Stage was according to the Barcelona Clinic Liver Cancer (BCLC } \\
\text { Staging System: Early HCC (Stages } 0 \& \text { A), Advanced (Stage B, C, D); LC Stage Was According To Child-Pugh Scoring System: Early LC (Child A); Late LC (Chil } \\
\text { B, C); P: Probability; P1: Compared early HCC and early AFP -ve HCC; P2: Compared early HCC and total HCC; P<0.05 was considered to be statisticall } \\
\text { significant; S: Significant; NS: Non-Significant. }\end{array}$} \\
\hline
\end{tabular}

Table 4: Spearman's rho Correlation between plasma levels of both CAP2 and AFP and the clinico-pathological characteristics in patients with HCC and LC.

\begin{tabular}{|c|c|c|c|c|c|c|c|c|}
\hline \multirow[t]{2}{*}{ Variables } & \multicolumn{2}{|c|}{ CAP2 HCC } & \multicolumn{2}{|c|}{ AFP HCC } & \multicolumn{2}{|c|}{ CAP2 LC } & \multicolumn{2}{|l|}{ AFP LC } \\
\hline & $r$ & $\mathrm{p}$ & $r$ & $\mathrm{p}$ & $r$ & $\mathrm{p}$ & $r$ & $\mathrm{p}$ \\
\hline AFP HCC & $-0.116-$ & 0.156 & -- & -- & -- & -- & -- & -- \\
\hline AFP LC & -- & -- & -- & -- & 0.078 & 0.528 & -- & -- \\
\hline Age & 0.131 & 0.111 & 0.274 & 0.060 & $-0.231-$ & 0.094 & 0.206 & 0.132 \\
\hline Gender & $-0.135-$ & 0.099 & 0.129 & 0.115 & $-0.202-$ & 0.285 & 0.009 & 0.917 \\
\hline Etiology & 0.041 & 0.622 & $-0.153-$ & 0.061 & $-0.065-$ & 0.427 & $-0.048-$ & 0.563 \\
\hline Tumor size & 0.294 & 0.000 & 0.224 & 0.056 & -- & -- & -- & -- \\
\hline Metastasis & 0.342 & 0.000 & 0.099 & 0.229 & -- & -- & -- & -- \\
\hline Portal vein thrombosis & 0.168 & 0.001 & 0.152 & 0.064 & -- & -- & -- & -- \\
\hline BCLC & 0.286 & 0.000 & 0.151 & 0.066 & -- & -- & -- & -- \\
\hline Child Pugh scores & 0.295 & 0.000 & 0.086 & 0.296 & 0.136 & 0.097 & 0.150 & 0.067 \\
\hline \multicolumn{9}{|c|}{$\begin{array}{l}\text { CAP2: Adenylyl Cyclase-Associated Protein-2 (ng/mL); HCC: Hepatocellular Carcinoma; LC: Liver Cirrhosis; AFP: Serum Alpha-Fetoprotein Level (ng/mL); -ve: } \\
\text { Negative; +ve: Positive; BCLC: Barcelona Clinic Liver Cancer Staging System; r: Spearman's rho Correlation Coefficient; P: Probability; P<0.05 was considered to } \\
\text { be statistically significant }\end{array}$} \\
\hline
\end{tabular}

CAP2 and AFP levels in All HCC patients (Table 5 and Figure 2A): CAP2 had a better diagnostic value than AFP as it had a significantly higher AUC $(95 \% \mathrm{Cl})$ at $0.86(0.79-0.93)$ vs. 0.75 (0.65-0.85), Sensitivity of $(81.5 \%$ vs. $62 \%)$, with a positive predictive value of $86.3 \%$ vs. $79.5 \%(P=0.001)$. Moreover, the combined diagnostic value of both (CAP2+AFP) was statistically significantly better than either CAP2 or AFP alone with highest AUC $(95 \% \mathrm{Cl})$ at $0.89(0.83-0.95)$ and Sensitivity of $87.8 \%$ $(P=0.012)$. 


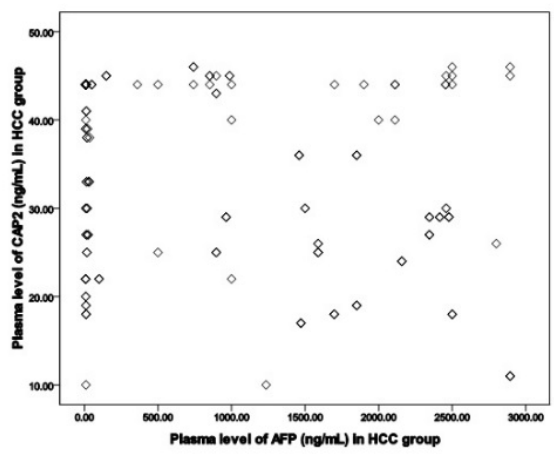

Figure 1: Correlation between the plasma levels of CyclaseAssociated Protein-2 (CAP2) and Alpha-fetoprotein (AFP) in Hepatocellular carcinoma (HCC) patients.

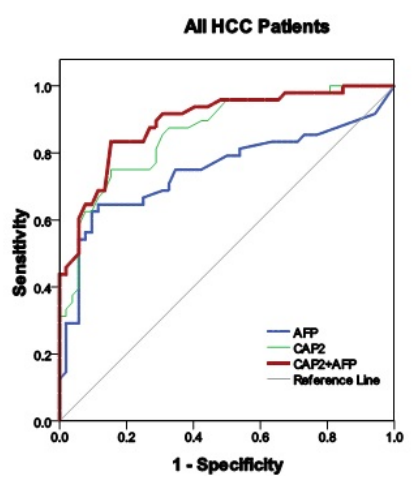

Figure 2A: The receiver operating characteristic (ROC) of Cyclase-Associated Protein-2 (CAP2) and Alpha-fetoprotein (AFP) in All HCC patients.

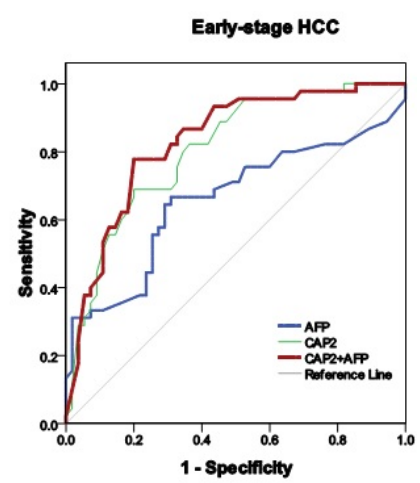

Figure 2B: The receiver operating characteristic (ROC) of Cyclase-Associated Protein-2 (CAP2) and Alpha-fetoprotein (AFP) in early-stage HCC patients.

CAP2 and AFP levels in Early-Stage HCC patients (Table 5 and Figure 2B): From all HCC patients only 68 patients had early-stage HCC. CAP2 had a better diagnostic value than AFP as it had a statistically significantly higher AUC $(95 \% \mathrm{CI})$ at 0.80
(0.72-0.89) vs. $0.68(0.58-0.79)$, Sensitivity of $(80.5 \%$ vs. $43.1 \%)$, Specificity of $81.3 \%$ vs. $79.9 \%$ with a positive predictive value of $82.2 \%$ vs. $64.7 \%$ ( $P=0.001)$. Moreover, the diagnostic value of combined (CAP2+AFP) was statistically significantly better than AFP alone with the highest AUC (95\% $\mathrm{Cl})$ at 0.82 (0.74-0.90), Sensitivity of $81.1 \%$ and Specificity of $80.4 \%$ with a positive predictive value of $81.8 \%(P=0.001)$. Nevertheless, the diagnostic value of combined (CAP2+AFP) was not significantly different from CAP2 alone $(P=0.053)$. The cutoff value for combined (CAP2+AFP) was CAP2 $(6.5 \mathrm{ng} / \mathrm{mL})$ or AFP (10 ng/mL).

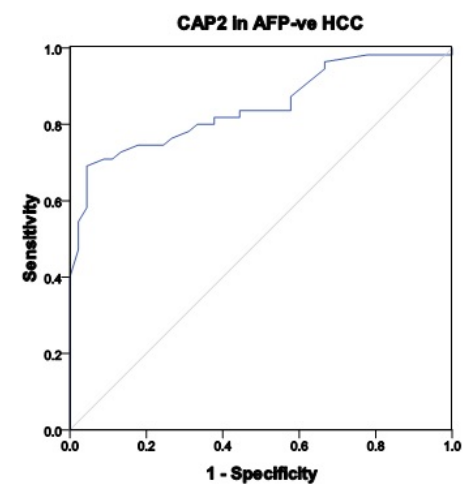

Figure 2C: The receiver operating characteristic (ROC) of Cyclase-Associated Protein-2 (CAP2) in Alpha-fetoproteinnegative HCC patients.

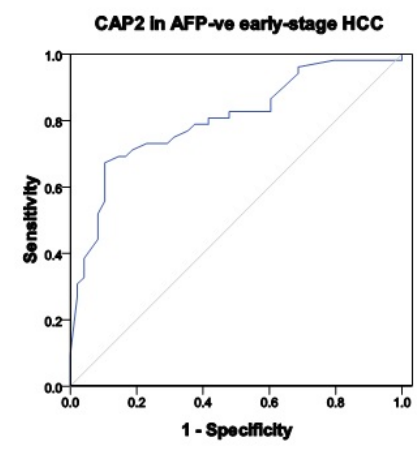

Figure 2D: The receiver operating characteristic (ROC) of Cyclase-Associated Protein-2 (CAP2) in Alpha-fetoproteinnegative early-stage HCC patients.

CAP2 levels in AFP-ve HCC patients (Table 5, Figures $2 \mathrm{C}$ and 2D): From all HCC patients only 56 patients had AFP-ve HCC, of which 50 patients had AFP-ve early-stage HCC. Using a cutoff value of $7.5 \mathrm{ng} / \mathrm{mL}$ when AFP levels were below $15 \mathrm{ng} / \mathrm{mL}$, CAP2 was found to have a good diagnostic value for AFP-ve HCC patients (Figure 2C) and AFP-ve Early-stage HCC patients (Figure 2D) with AUC $(95 \% \mathrm{Cl})$ at $0.85(0.77-0.92)$ and 0.80 (0.72-0.89) respectively, Sensitivity at $82.6 \%$ and $82.1 \%$ respectively, Specificity at $81.1 \%$ and $79.2 \%$ respectively and a 
positive predictive value at $85.1 \%$ and $84.6 \%$ respectively

(Table 5).

Table 5: Diagnostic cutoff values, AUC, Sensitivity, Specificity, and predictive values of CAP2 and AFP in HCC group and its subgroups.

\begin{tabular}{|c|c|c|c|c|c|c|c|c|c|c|}
\hline \multicolumn{2}{|c|}{ Variables } & \multirow{2}{*}{$\begin{array}{l}\text { AUC }(95 \% \mathrm{Cl}) \\
0.75 \\
(0.65-0.85)\end{array}$} & \multirow{2}{*}{$\begin{array}{l}\text { S. Error } \\
0.052\end{array}$} & \multirow{2}{*}{$\begin{array}{l}\begin{array}{l}\text { Cutoff value } \\
\text { (ng/mL) }\end{array} \\
15\end{array}$} & \multirow{2}{*}{$\begin{array}{l}\text { Sensitivity (\%) } \\
62\end{array}$} & \multirow{2}{*}{$\begin{array}{l}\text { Specificity (\%) } \\
83.9\end{array}$} & \multirow{2}{*}{$\begin{array}{l}\text { PPV } \\
\text { (\%) }\end{array}$} & \multirow{2}{*}{$\begin{array}{l}\begin{array}{l}\text { NPV } \\
(\%)\end{array} \\
68.7\end{array}$} & \multirow{2}{*}{$\begin{array}{l}\text { PLR } \\
3.9\end{array}$} & \multirow{2}{*}{$\begin{array}{l}\text { P-value } \\
0.001^{*}\end{array}$} \\
\hline $\begin{array}{l}\text { All } \\
\text { HCC }\end{array}$ & AFP & & & & & & & & & \\
\hline & CAP2 & $\begin{array}{l}0.86 \\
(0.79-0.93)\end{array}$ & 0.036 & 7.5 & 81.5 & 78.8 & 86.3 & 72.2 & 3.8 & $0.012^{* *}$ \\
\hline & $\begin{array}{l}\text { CAP2+ } \\
\text { AFP }\end{array}$ & $\begin{array}{l}0.89 \\
(0.83-0.95)\end{array}$ & 0.049 & $\begin{array}{l}\mathrm{CAP} 2=6.5 \text { or } \\
\mathrm{AFP}=10\end{array}$ & 87.8 & 76.7 & 86.7 & 78.6 & 3.8 & $0.001^{* * *}$ \\
\hline \multirow[t]{3}{*}{$\begin{array}{l}\text { Early } \\
\text { HCC }\end{array}$} & AFP & $\begin{array}{l}0.68 \\
(0.58-0.79)\end{array}$ & 0.053 & 15 & 43.1 & 79.9 & 64.7 & 63.3 & 2.2 & $0.001^{*}$ \\
\hline & CAP2 & $\begin{array}{l}0.80 \\
(0.72-0.89)\end{array}$ & 0.044 & 7.5 & 80.5 & 81.3 & 82.2 & 77.6 & 4.2 & $0.053^{\star *}$ \\
\hline & $\begin{array}{l}\text { CAP2+ } \\
\text { AFP }\end{array}$ & $\begin{array}{l}0.82 \\
(0.74-0.91)\end{array}$ & 0.042 & $\begin{array}{l}\text { CAP2 }=6.5 \text { or } \\
A F P=20\end{array}$ & 81.1 & 80.4 & 81.8 & 79.6 & 4.1 & $0.001^{* * *}$ \\
\hline $\begin{array}{l}\text { AFP-ve } \\
\text { HCC }\end{array}$ & CAP2 & $\begin{array}{l}0.85 \\
(0.77-0.92)\end{array}$ & 0.039 & 7.5 & 82.6 & 81.1 & 85.1 & 78.2 & 4.4 & - \\
\hline $\begin{array}{l}\text { AFP-ve } \\
\text { early } \\
\text { HCC }\end{array}$ & CAP2 & $\begin{array}{l}0.80 \\
(0.72-0.88)\end{array}$ & 0.044 & 7.5 & 82.1 & 79.2 & 84.6 & 76 & 3.9 & - \\
\hline
\end{tabular}

Abbreviations: AUC: Area Under The Curve; PPV: Positive Predictive Value; NPV: Negative Predictive Value; PLR: Positive Likelihood Ratio; CAP2: Adenylyl Cyclase-Associated Protein-2 (ng/mL); HCC: Hepatocellular Carcinoma; AFP: Serum Alpha-Fetoprotein Level (ng/mL); -ve: Negative; P: Probability ("AFP vs. CAP2; ${ }^{* *}$ CAP2 vs. Combined CAP2+AFP; ${ }^{* *}$ AFP vs. Combined CAP2+AFP); $P<0.05$ was considered to be statistically significant

\section{Discussion}

Hepatocellular carcinoma is an aggressive deadly primary hepatic cancer with far limited therapeutic strategies which are effective only at an early-stage of HCC. Unfortunately, alpha fetoprotein (AFP) has a limited performance and no longer recommended by international surveillance guidelines [30]. So, the discovery of an affordable non-invasive deployable biomarker with a high-performance and prognostic prediction for this aggressive disease has become a major focus of cancer research.

Recently, numerous biomarkers had been proposed for HCC diagnosis such as Glypican-3 (GPC3), Heat shock proteins (HSP70), Annexin A2 (ANXA2), microRNAs panel (miRNAs), Eag1 channels, Exosomes, Osteopontin (OPN), Neopterin and Cyclase-Associated Protein-2 (CAP2) [31]. The current study investigated CAP2 as a diagnostic biomarker for HCC patients in contrast to AFP and further evaluated its role in detecting early-stage and AFP-negative HCC Egyptian patients.

In contrast to previous studies [5], chronic Hepatitis C was the commonest etiology of liver cirrhosis in either HCC or nonHCC patients (63.3\% and $62 \%$ respectively) followed by chronic Hepatitis B (20\% and $18.7 \%$ respectively) however, there were no significant differences in plasma levels of either CAP2 or AFP related to chronic hepatitis $B$ or $C$. This may be explained by the high endemicity of chronic HCV in Egypt.

The current data revealed that HCC patients had a significantly higher plasma AFP and CAP2 levels than control and LC patients. Nevertheless, Early-stage HCC patients had no significant elevation in AFP levels. This finding could imply a role of CAP2 in the prediction of HCC and coincided with a previous study in which the authors proposed that plasma CAP2 level is a promising biomarker complementary to AFP in diagnosing HCC [32].

In human, CAP2 was detectable and differently expressed in many cancers being highly upregulated in thyroid, kidney, bladder, breast cancers [21]. However, there is paucity in literature describing the relevant prognostic implication of plasma levels of CAP2 in HCC patients.

In line with current data, Fu et al. suggested CAP2 as a promising biomarker for HCC prognosis as it noticeably overexpressed in HCC tissues, compared with the non-cancerous tissues and significantly correlated with poor survival. Of interest, they proposed the prognostic implication of CAP2 in the subgroups of HCC patients [33].

The current study also demonstrated that the higher plasma levels of CAP2 in HCC patients were significantly positively correlated with disease severity, BCLC stages of HCC, tumor nodules, the presence of either metastasis or portal vein thrombosis $(\mathrm{P}=0.001)$ but not with age, gender or offending etiology of LC or HCC. Moreover, high plasma CAP2 levels were significantly associated with high Child Pugh scores in HCC patients with underlying cirrhosis. Nevertheless, there were no significant correlations between plasma levels of CAP2 and AFP in HCC patients. The results presented in this study could also imply a prognostic role of plasma CAP2 level in HCC 
patients as well as HCC subgroups. Alpha fetoprotein (AFP) which was the golden marker of HCC lacks both sensitivity and specificity and has limited performance especially in earlystage HCCs and is no longer recommended by international surveillance guidelines [30].

Despite the advanced imaging methods, it is still difficult to detect early-stage HCC and AFP-ve HCC patients. Recently, numerous biomarkers had been proposed to predict earlystage HCC as well as AFP-negative HCC patients such as desgamma-carboxy prothrombin (DCP) (also known as Prothrombin Induced by Vitamin K Absence II: PIVKA II), Lens culinaris-agglutinin-reactive fraction of AFP (AFP-L3) [34], Glypican-3 (GPC3) [35], fucosylated haptoglobin [36], fucosylated paraoxonase 1 (FUC-PON1) [37]; Heat shock proteins (HSP70) [38], Annexin A2 (ANXA2) [39], microRNAs panel (miRNAs) [40], Eag1 channels [41], Transforming Growth Factor-Beta [42], Osteopontin (OPN) [43] and CAP2 [32,33]. The combination of PIVKA-II, the sensitivity of which was $48.9 \%$ in HCC patients, with AFP or AFP-L3 significantly improved its diagnostic performance [34]. Haptoglobin which had an AUC at $0.76 \%$, Sensitivity at $72.2 \%$ and Specificity at $70 \%$ was reported to complement AFP diagnostic performance [36].

Chen et al. suggested that CAP2 has a pivotal and superior role in differentiating HCC from LC being had a better sensitivity at $82.6 \%$ vs. $59.3 \%$, higher AUC at 0.86 vs. 0.75 than AFP [32]. This evidence coincides with the current study in which, CAP2 had a better diagnostic value than AFP, a significantly higher AUC $(95 \% \mathrm{Cl})$ at $0.86(0.79-0.93)$ vs. 0.75 (0.65-0.85), a significantly higher Sensitivity of ( $81.5 \%$ vs. $62 \%)$, and a higher positive predictive value of $86.3 \%$ vs. $79.5 \%$. Moreover, the combined diagnostic value of both (CAP2+AFP) in HCC patients was statistically significantly better than either CAP2 or AFP alone with highest AUC $(95 \% \mathrm{Cl})$ at 0.89 (0.83-0.95) and Sensitivity of $87.8 \%$. From the present data, it is evident that CAP2 alone or combined with AFP had higher AUC, Sensitivity, positive predictive value and overall diagnostic accuracy than those mentioned in other studies for CAP2 or for other biomarkers.

Additionally, the observed absence of a significant correlation between plasma levels of CAP2 and AFP in HCC patients supported the diagnosis of early-stage HCC, AFP -ve HCC, and AFP -ve early-stage HCC patients. Moreover, the diagnostic value of combined (CAP2+AFP) in detecting earlystage HCC was statistically significantly better than AFP alone with higher AUC $(95 \% \mathrm{Cl})$ at $0.82(0.74-0.90)$, Sensitivity of $81.1 \%$ and Specificity of $80.4 \%$ with a positive predictive value of $81.8 \%$. Using a cutoff value of $7.5 \mathrm{ng} / \mathrm{mL}$ when AFP levels were below $15 \mathrm{ng} / \mathrm{mL}$, CAP2 was found to have a high diagnostic accuracy for both AFP-ve HCC patients and AFP-ve early-stage $\mathrm{HCC}$ patients with AUC $(95 \% \mathrm{Cl})$ at $0.85(0.77-0.92)$ and $0.80(0.72-0.89)$ respectively, Sensitivity at $82.6 \%$ and $82.1 \%$ respectively, Specificity at $81.1 \%$ and $79.2 \%$ respectively and a positive predictive value at $85.1 \%$ and $84.6 \%$ respectively.

The mechanism of increased plasma CAP2 levels in HCC is still not clear. Shibata and colleagues evidenced that CAP2 was involved in and related to hepatic carcinogenesis being noticeably over-expressed and upregulated in HCC cells demonstrated by remarkably higher immunohistochemistry (IHC) Score. On the other hand, no or trivial CAP2 expression in normal hepatocytes was noticed [44]. In-vivo and in-vitro immunohistochemistry (IHC) staining showed that CAP2 was distributed in the nuclear and perinuclear areas suggesting that CAP2 might be produced by HCC cells of differentiated as well as early-stage HCC [22]. CAP is involved in regulating the adenylyl cyclase activity in yeast under the control of RAS. CAP2 is released from the nucleus by high salt concentrations and released into blood stream by vesicles formation and trafficking [45]. However, the detailed mechanisms for CAP2 release into the blood stream still elusive and necessitate future studies.

In this study, CAP2 had a better diagnostic value than AFP in detecting Early-stage HCC; with higher AUC $(95 \% \mathrm{Cl})$ at 0.80 (0.72-0.89) vs. $0.68(0.58-0.79)$, Sensitivity of $(80.5 \%$ vs. $43.1 \%)$, Specificity of $81.3 \%$ vs. $79.9 \%$ and a positive predictive value of $82.2 \%$ vs. $64.7 \%$. Additionally, the plasma CAP2 level was higher in early-stage HCCs than in LC patients. Consequently, it could be proposed for Early-stage HCC diagnosis and distinguishing AFP-negative Early-stage HCC from LC.

In similar studies, some novel biomarkers were also proposed for Early-stage HCC diagnosis and differentiate AFPnegative Early-stage HCC from LC [46]. Fucosylated Paraoxonase 1 (Fuc-Pon1) was proposed as a promising biomarker for diagnosis of early-stage $\mathrm{HCC}$ and distinguishing AFP-negative early-stage HCC from LC patients [37]. Osteopontin (OPN) alone or in combination with AFP provided a significantly AUC, higher sensitivity, overall accuracy and performance than AFP in diagnosing LC, HCCs, early-stage HCC and AFP-negative HCC patients [43]. Fucosylated Haptoglobin (Hp) /Haptoglobin ratio alone or combined with AFP and the ELISA Index (optical density [OD] value of fucosylated $\mathrm{Hp} / \mathrm{OD}$ value of $\mathrm{Hp}$ ) had a better diagnostic performance in Early-stage HCC and AFP-negative HCC [36].

Similarly, the serum level of Annexin A2 (ANXA2) might be a good biomarker for detection of early of HCC and could differentiate between HCC and CLD as it is over-expressed and upregulated in HCC cells [47]. Unfortunately, the diagnostic performance of serum Glypican 3 (GPC3) which had a diagnostic value comparable to AFP in HCC diagnosis is still unsatisfactory for early-stage HCC [48]. However, both serum GPC-3 and GPC-3mRNA are promising diagnostic markers for early detection of HCC in Egyptian patients $[35,49]$. The diagnostic accuracy and performance of plasma CAP2 levels for early-stage HCC noted in the current study were much higher than that of previously studied biomarkers (such as AFP-L3, PIVKA II, GPC3, HSP70, OPN, FUC-PON1, ANXA2). Meanwhile, CAP2 was found to have a high diagnostic accuracy for both AFP-ve HCC patients and AFP-ve early-stage $\mathrm{HCC}$ patients with AUC $(95 \% \mathrm{Cl})$ at $0.85(0.77-0.92)$ and 0.80 (0.72-0.89) respectively, Sensitivity at $82.6 \%$ and $82.1 \%$ respectively, Specificity at $81.1 \%$ and $79.2 \%$ respectively and a positive predictive value at $85.1 \%$ and $84.6 \%$ respectively. 
Collectively, we could suggest that CAP2 might be a superior biomarker for detection of early-stage HCC, AFP-ve HCC patients and AFP-ve Early-stage HCC patients. Also, the combined diagnostic value of both CAP2+AFP (AUC: 0.89 (0.83-0.95)) in HCC patients was statistically significantly better than either CAP2 (AUC: $0.86(0.79-0.93)$ ) or AFP (AUC: 0.75 (0.65-0.85)) alone with the highest Sensitivity at $87.8 \%$ vs. $86.3 \%$ or $83.9 \%$ for CAP2 or AFP respectively. In addition, combined CAP2+AFP could greatly improve the detection rate of all HCCs and differentiate early-stage of HCC from liver cirrhosis. Nevertheless, the diagnostic value of the combined CAP2+AFP in early-stage HCCs was not significantly different from CAP2 alone [AUC: 0.82 (0.74-0.91 vs. 0.80 (0.72-0.89)].

To determine the factors that may affect the plasma CAP2 level, we further investigate if plasma CAP2 level is related to the clinico-pathological parameters of the tumor. The clinicopathological features of HCC patients such as tumor size, histological grade, metastasis, portal vein thrombosis, BCLC stage and clinical stage were proposed to be relevant prognostic factors of tumor progression [50]. Effendi et al. demonstrated that CAP2 levels were associated with the clinicopathological features of HCC patients [51]. Similarly, we found significant positive correlations between plasma CAP2 level and clinico-pathological features of HCC patients such as tumor size, BCLC stage, metastasis, portal vein thrombosis, and clinical stage. Zhang et al. also, suggested that BCLC stage B and $C$ were associated with poor overall survival and tumor recurrence in AFP-ve HCC patients [52]. Taken together, CAP2 levels might be a predictor for HCC. However, this assumption should be validated by future large-scale surveillance studies.

\section{Conclusion}

In conclusion, plasma CAP2 levels alone or combined with AFP could be a promising superior biomarker for diagnosis of $\mathrm{HCC}$, detection of early-stage of HCC, prediction of AFPnegative $\mathrm{HCC}$ and differentiating it from liver cirrhosis.

\section{Conflict of Interest}

The authors declare no conflicts of interest

\section{Acknowledgement}

Authors thank Prof. Dr. Salah El-Gamal, and Dr. Aya Mohammed Amin for their support and help.

\section{References}

1. El-Serag HB (2012) Epidemiology of viral hepatitis and hepatocellular carcinoma. Gastroenterology 142: 1264-1273.

2. International Agency for Research on Cancer (2014) World cancer report 2014. Geneva: WHO, 2014.

3. El-Serag HB (2011) Hepatocellular carcinoma. N Engl J Med 365: 1118-1127.

4. El-Houseini ME, Mohammed MS, Elshemey WM, Hussein TD, Desouky OS, et al. (2005) Enhanced detection of hepatocellular carcinoma. Cancer Control 12: 248-253.
5. Sanyal AJ, Yoon SK, Lencioni R (2010) The etiology of hepatocellular carcinoma and consequences for treatment. Oncologist 15: 14-22.

6. Allemani C, Weir HK, Carreira H, Harewood R, Spika D, et al. (2015) Global surveillance of cancer survival 1995-2009: analysis of individual data for 25,676,887 patients from 279 population-based registries in 67 countries (CONCORD-2). Lancet 385: 977-1010.

7. Llovet JM, Zucman-Rossi J, Pikarsky E, Sangro B, Schwartz M, et al. (2016) Hepatocellular carcinoma. Nat Rev Dis Primers 2: 16018.

8. Torre LA, Bray F, Siegel RL, Ferlay J, Lortet-Tieulent J, et al. (2015) Global cancer statistics, 2012. CA Cancer J Clin 65: 87-108.

9. Liu Y, Hong Z, Tan G, Dong X, Yang G, et al. (2014) NMR and LC/MS-based global metabolomics to identify serum biomarkers differentiating hepatocellular carcinoma from liver cirrhosis. Int J Cancer135: 658-668.

10. Giannini EG, Marenco S, Borgonovo G, Savarino V, Farinati F, et al. (2012) Alpha-fetoprotein has no prognostic role in small hepatocellular carcinoma identified during surveillance in compensated cirrhosis. Italian Liver Cancer (ITALICA) group. Hepatology 56: 1371-1379.

11. Kim JU, Shari MIF, Crossey MME, Gomez-Romero M, Holmes E, et al. (2016) Hepatocellular carcinoma: Review of disease and tumor biomarkers. World Journal of Hepatology 8: 471-484.

12. Tunissiolli NM, Castanhole-Nunes MMU, Biselli-Chicote PM, Pavarino EC, Da Silva RF, et al. (2017) Hepatocellular Carcinoma: A Comprehensive Review of Biomarkers, Clinical Aspects, and Therapy. Asian Pac J Cancer Prev 18: 863-872.

13. El-Tayeh SF, Hussein TD, El-Houseini ME, Amer MA, El-Sherbini $M$, et al. (2012) Serological biomarkers of hepatocellular carcinoma in Egyptian patients. Dis Markers 32: 255-263.

14. Wang CH, Wey KC, Mo LR, Chang KK, Lin RC, et al. (2015) Current trends and recent advances in diagnosis, therapy and prevention of hepatocellular carcinoma. Asian Pac J Cancer Prev 16: 3595-3604.

15. Dodatko T, Fedorov AA, Grynberg M, Patskovsky Y, Rozwarski DA, et al. (2004) Crystal structure of the actin binding domain of the cyclase-associated protein. Biochemistry 33: 10628-10641.

16. Peche V, Shekar S, Leichter M, Korte H, Schroder R, et al. (2007) CAP2, cyclase-associated protein 2, is a dual compartment protein. CellMol Life Sci 64: 2702-2715.

17. Field J, Vojtek A, Ballester R, Bolger G, Colicelli J, et al. (1990) Cloning and characterization of CAP, the $S$. cerevisiae gene encoding the $70 \mathrm{kd}$ adenylyl cyclase-associated protein. Cell 61: 319-327

18. Swiston J, Hubberstey A, Yu G, Young D (1995) Differential expression of CAP and CAP2 in adult rat tissues. Gene 165: 273277.

19. Shima F, Okada T, Kido M, Sen H, Tanaka Y, et al. (2000) Association of yeast adenylyl Cyclase with Cyclase-associated protein CAP forms a second Ras-binding site which mediates its Ras-dependent activation. Mol Cell Biol 20: 26-33.

20. Peche VS, Holak TA, Burgute BD, Kosmas K, Kale SP, et al. (2013) Ablation of cyclase-associated protein 2 (CAP2) leads to cardiomyopathy. Cell Mol Life Sci 70: 527-543. 
21. Xu L, Peng S, Huang Q, Liu Y, Jiang H, et al. (2016) Expression status of Cyclase-associated protein 2 as a prognostic marker for human breast cancer. Oncology Reports 36: 1981-1988.

22. Xie YY, Xu QX, Han SY, Zhang LD, Bai YQ, et al. (2010) The expression and significance of adenylate Cyclase-associated protein 2 in human hepatocellular carcinoma. Zhonghua Gan Zang Bing Za Zhi 18: 546-547.

23. Peng Y, Qi X, Guo X (2016) Child-Pugh Versus MELD Score for the assessment of prognosis in liver cirrhosis: A systematic review and meta-analysis of observational studies. Fan $\mathrm{H}$ ed. Medicine. 95: e2877.

24. Goodman ZD (2007) Grading and staging systems for inflammation and fibrosis in chronic liver diseases. J Hepatol 47: 598-607.

25. Liu PH, Hsu CY, Hsia CY, Lee YH, Chiou YY, et al. (2017) ALBI and PALBI grade predict survival for HCC across treatment modalities and BCLC stages in the MELD Era. J Gastroenterol Hepatol 32: 879-886.

26. Bruix J, Sherman M (2011) American Association for the Study of Liver Diseases; Management of hepatocellular carcinoma: An update. Hepatology 53: 1020-1022.

27. Duseja A (2014) Staging of hepatocellular carcinoma. Journal of Clinical and Experimental Hepatology 4: S74-S79.

28. Dalton LA (2016) Optimal ROC-based classification and performance analysis under Bayesian uncertainty models, IEEE/ACM Transactions on Computational Biology and Bioinformatics (TCBB) 13: 719-729.

29. Mercaldo ND, Lau KF, Zhou XH (2007) Confidence intervals for predictive values with an emphasis to case-control studies. Statistics in Medicine 26: 2170-2183.

30. Cai L, Rao X, Su Q, Qin J, Cai LQ, et al. (2015) Diagnostic value of Joint Detection of GP73 and AFP-L3 in Primary Hepatic Carcinoma with Low Concentration of AFP. J Int Transl Med 3: 28-32.

31. Song P, Tang Q, Feng X, Tang W (2016) Biomarkers: evaluation of clinical utility in surveillance and early diagnosis for hepatocellular carcinoma. Scand J Clin Lab Invest Suppl 245: S70-S76.

32. Chen M, Zheng T, Han S, Zhang L, Bai Y, et al. (2015) A preliminary study of plasma cyclase-associated protein 2 as a novel biomarker for early-stage and alpha-fetoprotein negative hepatocellular carcinoma patients. Clin Res Hepatol Gastroenterol 39: 215-221.

33. Fu J, Li M, Wu DC, Liu LL, Chen SL, et al. (2015) Increased expression of CAP2 indicates poor prognosis in hepatocellular carcinoma. Translational Oncology 8: 400-406.

34. Park SJ, Jang JY, Jeong SW, Cho YK, Lee SH, et al. (2017) Usefulness of AFP, AFP-L3, and PIVKA-II, and their combinations in diagnosing hepatocellular carcinoma. Medicine 96: e5811.

35. Abdelgawad IA, Mossallam GI, Radwan NH, Elzawahry HM, Elhifnawy NM (2013) Can Glypican3 be diagnostic for early hepatocellular carcinoma among Egyptian patients? Asian Pac J Cancer Prev 14: 7345-7349.

36. Shang S, Li W, Qin X, Zhang S, Liu Y (2017) Aided diagnosis of hepatocellular carcinoma using serum fucosylated haptoglobin ratios. Journal of Cancer 8: 887-893.
37. Shu H, Li W, Shang S, Qin X, Zhang S, et al. (2017) Diagnosis of AFP-negative early-stage hepatocellular carcinoma using FucPON1. Discov Med 23: 163-168.

38. Wang C, Zhang Y, Guo K, Wang N, Jin H, et al. (2016) Heat shock proteins in hepatocellular carcinoma: Molecular mechanism and therapeutic potential. Int J Cancer 138: 1824-1834.

39. Shaker MK, Abdel Fattah HI, Sabbour GS, Montasser IF, Abdelhakam SM, et al. (2017) Annexin A2 as a biomarker for hepatocellular carcinoma in Egyptian patients. World Journal of Hepatology 9:469-76.

40. Hayes CN, Chayama K (2016) MicroRNAs as biomarkers for liver disease and hepatocellular carcinoma. Int J Mol Sci 17: 280.

41. Chávez-López MG, Zúñiga-García V, Pérez-Carreón Jl, AvalosFuentes A, Escobar Y, et al. (2016) Eag1 channels as potential early-stage biomarkers of hepatocellular carcinoma. Biologics: Targets and Therapy 10: 139-148.

42. Mansour AH, Elkhodary TR, Anwar R, Habeeb MR, Mohammed MA (2014) Regulation of cancer stem cell marker (CD133) by transforming growth factor beta in hepatocellular carcinoma. International Journal of Cancer Research 10: 65-73.

43. Shang S, Plymoth A, Ge S, Feng Z, Rosen HR, et al. (2012) Identification of osteopontin as a novel marker for early hepatocellular carcinoma. Hepatology 55: 483-490.

44. Shibata R, Mori T, Du W, Chuma M, Gotoh M, et al. (2006) Overexpression of cyclase-associated protein 2 in multistage hepatocarcinogenesis. Clin Cancer Res 12: 5363-5368.

45. Zelicof A, Protopopov V, David D, Lin XY, Lustgarten V, et al. (1996) Two separate functions are encoded by the carboxylterminal domains of the yeast Cyclase-associated protein and its mammalian homologs. Dimerization and actin binding. J Biol Chem 271: 18243-18252.

46. Lou J, Zhang L, Lv S, Zhang C, Jiang S (2017) Biomarkers for hepatocellular carcinoma. Biomarkers in Cancer 9: 1-9.

47. Mohammad HS, Kurokohchi K, Yoneyama H, Tokuda M, Morishita A, et al. (2008) Annexin A2 expression and phosphorylation are up-regulated in hepatocellular carcinoma. Int J Oncol 33: 1157-1163.

48. Jia X, Liu J, Gao Y, Huang Y, Du Z (2014) Diagnosis accuracy of serum glypican-3 in patients with hepatocellular carcinoma: $A$ systematic review with meta-analysis. Arch Med Res 45: 580-588.

49. Sun B, Huang Z, Wang B, Yu Y, Lin S, et al. (2017) Significance of glypican-3 (GPC3) expression in hepatocellular cancer diagnosis. Med Sci Monit 23: 850-855.

50. Hayes DF, Isaacs C, Stearns V (2001) Prognostic factors in breast cancer: Current and new predictors of metastasis. J MammaryGland Biol Neoplasia 6: 375-392.

51. Effendi K, Yamazaki K, Mori T, Masugi Y, Makino S, et al. (2013) Involvement of hepatocellular carcinoma biomarker, cyclaseassociated protein 2 in zebrafish body development and cancer progression. Exp Cell Res 319: 35-44.

52. Zhang XF, Qi X, Meng B, Liu C, Yu L, et al. (2010) Prognosis evaluation in alpha-fetoprotein negative hepatocellular carcinoma after hepatectomy: Comparison of five staging systems. Eur J Surg Oncol 36: 718-724. 